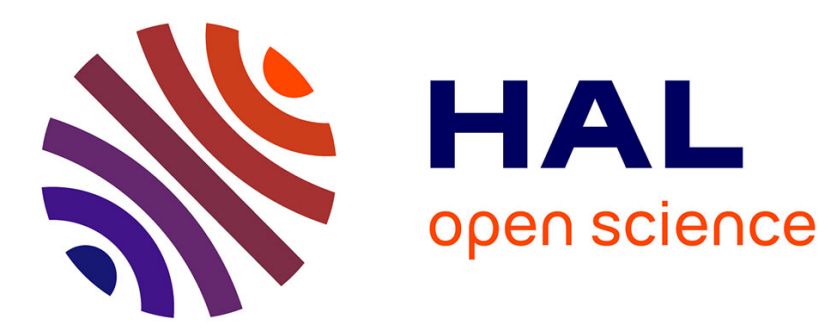

\title{
De la légende noire à l'assomption romantique. Le Cymbalum mundi entre XVIIe et XIXe siècles
}

\author{
Alain Mothu
}

\section{To cite this version:}

Alain Mothu. De la légende noire à l'assomption romantique. Le Cymbalum mundi entre XVIIe et XIXe siècles. Dix-septième siècle, 2019, n²83 (2), pp.211. 10.3917/dss.192.0211 . hal-02372423

\section{HAL Id: hal-02372423 \\ https://hal.sorbonne-universite.fr/hal-02372423}

Submitted on 20 Nov 2019

HAL is a multi-disciplinary open access archive for the deposit and dissemination of scientific research documents, whether they are published or not. The documents may come from teaching and research institutions in France or abroad, or from public or private research centers.
L'archive ouverte pluridisciplinaire HAL, est destinée au dépôt et à la diffusion de documents scientifiques de niveau recherche, publiés ou non, émanant des établissements d'enseignement et de recherche français ou étrangers, des laboratoires publics ou privés. 


\section{De la légende noire à l'assomption romantique \\ Le Cymbalum mundi entre XVII et XIX ${ }^{e}$ siècles}

Depuis sa prohibition précoce en 1538 jusqu'au début du XVIII siècle, où il ressortira des limbes à la faveur de sa redécouverte à la Bibliothèque royale puis de sa réédition en 1711 par Prosper Marchand (Amsterdam), le Cymbalum mundi connaît une occultation presque totale. Significativement, François Garasse n'en dit mot dans sa Doctrine curieuse des beaux esprits de ce temps ou prétendus tels (1623) et Bayle, à l'orée du XVIII ${ }^{\mathrm{e}}$ siècle, avoue n'avoir jamais vu cet ouvrage réputé «très-impie ${ }^{1}$. Bernard de La Monnoye, dix ans plus tard, parlera carrément de « résurrection ${ }^{2} »$. Autant dire qu'au XVII siècle, pour l'écrasante majorité des lettrés (nous en verrons les rares exceptions), le Cymbalum n'est plus qu'une rumeur ou une légende, placée sur le même pied que l'anonyme De tribus impostoribus et l'Ars nihil credendi prêté à Geoffroy Vallée - ces autres abominations «athées » surgies des Enfers qui ne survivent plus guère que dans l'imaginaire effaré des défenseurs de la foi ${ }^{3}$.

Un bilan s'impose. À la fin du XVII siècle, comme déjà un siècle plus tôt, les mieux informés croient alors savoir que l'ouvrage sortit des presses en 1537 ou en 1538 : François Grudé de La Croix du Maine, dans sa Bibliothèque (1584), avait formellement consigné la première date, quand Antoine Du Verdier, dans sa propre Bibliothèque (1585), avait décrit de manière détaillée un exemplaire lyonnais de 1538. Ni l'un ni l'autre n'indiquaient hélas leurs sources, mais nous savons que tous deux avaient raison, le Cymbalum parut premièrement à Paris, chez Jean Morin, sous la date de 1537 (la question de savoir en quel style interpréter cette date reste controversée), puis à Lyon, chez Benoît Bonyn, sous celle de $1538^{4}$.

$\mathrm{Si}$ nos érudits de la fin du XVII ${ }^{\mathrm{e}}$ peuvent également savoir que le Cymbalum, à la différence du De tribus impostoribus et de l'Ars nihil credendi, est inscrit depuis longtemps à l'Index (Sorbonne, 1544 ; Rome, 1559), ils ignorent tout de la procédure juridique qui le frappa en 1538, en même temps que le libraire Morin. Ils savent en revanche qu'il était écrit en français, et non en latin, mais ils supposent généralement, cette fois à tort, qu'il procédait effectivement d'une traduction latine : le subterfuge de Des Périers fonctionnait donc, qui, à l'ouverture de ses dialogues, donnait à lire une lettre de son soi-disant inventeur et traducteur, un certain Thomas du Clevier (soit Thomas incrédule, au v/n ou $\mathrm{u} / \mathrm{n}$ près: coquille typographique peut-être intentionnelle), à son ami Pierre Tryocan (soit Pierre croyant). Le premier aurait déniché l'original latin dans une lointaine bibliothèque orientale, à Dabas (ville

1 C'est dans la seconde édition du Dictionnaire historique et critique qu'apparaît pour la première fois l'article «Periers (Bonavanture des) » (Rotterdam, R. Leers, 1701, datée 1702, t. IV, p. 2380-2381).

2 «Lettre à M. Bouhier [...] sur le prétendu livre des trois Imposteurs » [16 juin 1712], dans Menagiana, $3^{\mathrm{e}}$ éd., Paris, F. Delaulne, 1715, IV, p. 283-312, ici p. 311.

3 Un De tribus impostoribus manuscrit, de conception apparemment récente commence à circuler timidement en Allemagne dans le dernier quart du siècle (selon Winfried Schröder, éd. du De imposturis religionum [De tribus impostoribus], Stuttgart-Bad Cannstatt, Frommann-Holzboog, 1999), mais nous n'avons identifié aucun manuscrit de La Beatitude des chrestiens (titre véritable de l'ouvrage de Vallée) et du Cymbalum mundi [dorénavant : $C M$ ] antérieur au XVIII siècle. Des ersatz manuscrits de ces derniers - «pseudo-Vallée » et «pseudo-Des Périers »- feront néanmoins leur apparition au tout début du XVIII ${ }^{\mathrm{e}}$ s., peu avant la redécouverte des originaux. Voir notre étude, «La Beatitude des chrestiens et son double clandestin », dans A. McKenna et A. Mothu (dir.), La Philosophie clandestine à l'Âge classique, Paris, Universitas, 1997, p. 79-117. Felix Frank, éditeur du Cymbalum en 1873 (Paris, Lemerre), fut apparemment le premier à signaler en France, d'après Vogt, l'existence du pseudo-Cymbalum manuscrit (p. LX).

4 La Croix du Maine, Bibliothèque («Premier volume»), Paris, L’Angelier, 1584, s.v. « Bonnadventure des Periers », p. 36-37 («il est autheur d'un livre destestable \& remply d'impietez intitulé Cymbalum Mundi, ou clochette du Monde, escrit premierement en latin par iceluy des Periers, \& depuis traduit par luy-mesme en François sous le nom de Thomas du Clevier, imprimé à Paris l'an 1537...»); Antoine Du Verdier, Bibliotheque, Lyon, Barthelemy Honorat, 1585, s.v. « Thomas du Clevier », p. 1177-1178. 
introuvable qui fait penser à Damas). Ainsi est-ce sous l'entrée «Thomas du Clevier » que Du Verdier nous parle du Cymbalum qu'il a sous les yeux. Et si La Croix du Maine se montre sur ce point mieux informé, attribuant le libelle à «Bonnadventure des Periers », autrefois «valet de chambre de de la Royne de Navarre sœur du Roy François I. », auteur connu par une poignée d'autres ouvrages dont «quelques contes \& faceties plaisantes, imprimees soubs son nom, soubs le tiltre de Nouvelles recreations ${ }^{5} »$, il croit toujours que le Cymbalum, ou «Clochette du Monde », fut « escrit premierement en Latin par iceluy des Periers, \& depuis traduit par luy-mesme en François soubs le nom de Thomas du Clevier ${ }^{6} »$. D'autres, comme Mersenne, dont nous reparlerons, n'attribueront à Des Périers que la traduction.

En tout cas, Du Verdier est un isolé : tous ceux qui ont lu, compulsé ou entendu parler de l'Apologie sur Hérodote d'Henri Estienne (1566), rééditée une dizaine de fois jusqu'en 1607, savent que Des Périers est impliqué dans l'édition du Cymbalum mundi et presque tout le monde le regarde positivement comme son auteur ${ }^{7}$. Ils ont appris dans la même source ou chez ses suiveurs, majoritairement protestants comme lui et déjà avertis par Calvin et Farel de la nocivité de «Deperius ${ }^{8}$ », que dans un accès de désespoir ou de folie, Bonaventure s'«enferr(a) de son espee sur laquelle il s'estoit jetté, l'ayant appuyee le pommeau contre terre, que la pointe entree par l'estomach sortoit par l'eschine ${ }^{9} »$. Ce récit, qui concorde si bien avec le topos apologétique de la mort providentiellement tragique ou piteuse de l'athée ${ }^{10}$, fut adopté par tout le monde : Chassanion (1581 et 1586), La Croix du Maine (1584), plus

5 Néanmoins ces contes, selon lui, seraient pour la plupart attribuables, à Jacques Peletier du Mans et Nicolas Denisot (ils en sont déclarés «les deux premiers autheurs ») : affirmation qu'a rejetée la critique moderne. Elle attribue bien à Des Périers les 90 contes publiés en 1558 sous le titre de Nouvelles Recreations et Joyeux Devis de feu Bonaventure Des Perriers (voir l'éd. de référence procurée par Krystina Kasprzyk, Paris, STFM, 1997).

${ }^{6}$ La Croix du Maine, op. cit., p. 36-37.

${ }^{7}$ Après s'être exclamé au chap. XVI : « qui ne sçait quel contempteur \& mocqueur de Dieu a esté Bonaventure des Periers, \& quels tesmoignages il en a rendu par ses livres ? », Estienne désigne à deux reprises, aux chap. XVIII et XXVI, «Bonaventure Des Periers, l'auteur du detestable livre intitulé cymbalum mundi»: Introduction au traité de la conformité des merveilles anciennes avec les modernes, ou Traité préparatif à l'Apologie pour Hérodote, s.l. [Genève, François Estienne], 1566 [éd. en 680 p.], L. I, p. 120, 309 et 414. Voir l'éd. récente donnée par Bénédicte Boudou : Traité preparatif..., Genève, Droz, 2007, I, p. 292, 506507 et 636, avec la Bibliographie p. 50 sq.

8 Tous deux stigmatisaient en 1550 l'aveuglement impie de «Deperius » sans mentionner le CM : Calvin, Des scandales, Genève, Jean Crespin, 1550, p. 74 (éd. O. Fatio, Génève, Droz, 1984, p. 138 ; De scandalis quibus hodie plerique absterrentur..., Genève, Crispin, 1550, p. 53) ; Farel, Le Glaive de la parolle veritable..., Genève, Jean Girard, 1550, p. 43 (les «erreurs des Libertins, mais aussi du tresméchant Deperius et des Atheistes »). Tout à l'inverse, les catholiques Postel (De Alcorani seu legis Mahometi et Evangelistrarum Concordia Liber, Paris, Gromorsus, 1543, p. 72) ou Pasquier (cf. infra, n. 14), accableront le CM sans en nommer l'auteur. Dans le cas de Postel, nous présumons qu'il l'ignorait (dans un texte manuscrit de 1560, il évoque des auteurs au pluriel, primi Cymbali Mundi auctores: voir C. Gilly, «Guillaume Postel et Bâle. Quelques additions à la Bibliographie des manuscrits de Guillaume Postel », dans Guillaume Postel, 15811981, Paris, Guy Trédaniel, 1985, p. 41-77, ici p. 71 ; et notre note « Combien de cymbales pour combien de musiciens » dans La Lettre clandestine, 26, 2018, p. 173-178).

${ }^{9}$ Henri Estienne, Introduction au traité, op. cit., p. 309 et 414 (éd. Boudou, p. 507 et 636). Il s'enferra ainsi, nous précise-t-on, «nonobstant la peine qu'on prenoit à le garder (à cause qu'on le voyoit estre desesperé, \& en deliberation de se desfaire). »

10 Topos que l'on fait communément remonter au Chronicon de saint Jérôme, quand celui-ci déclare que Lucrèce, devenu fou après avoir absorbé un philtre amoureux, se serait ensuite suicidé. La remarque n'avait pourtant rien d'infâmant dans l'esprit de Jérôme, selon Pierre Vesperini, Lucrèce. Archéologie d'un classique européen, Paris, Fayard, 2017, p. 195 sq. Il reste que cette folie et ce suicide furent durablement interprétés comme des marqueurs d'athéisme, en vertu du Dixit insipiens in corde suo non est Deus psalmique (Ps. XIV, 1 et LIII, 1) et du postulat que cette folie qui éteint toute crainte de la mort peut le conduire au « défi suprême » envers Dieu (cf. François Berriot, Athéismes et athéistes en France au XVI siècle, Lille, ANRT; Paris, Éditions du Cerf, [1984], p. 194), étant entendu que ce geste terrible est aussi commandé par le désespoir d'une vie sans Dieu et révèle un châtiment céleste. 
tard Mersenne (1623), Colletet (avant 1659), Catherinot (1685), Bayle (1702) et d'autres, mais surtout Simon Goulart, au chapitre des «Cas estranges, horribles, \& extremement pitoyables » de ses célèbres Histoires admirables et memorables de nostre temps (1603): Goulart retient d'Estienne que Des Périers «se mocque de la vraye religion, autant et plus que des superstitions » et il reprend son récit presque mot à mot $^{11}$. Prosper Marchand sera apparemment le premier à douter du suicide de Des Périers.

Notons en passant que personne ne se risquait alors à dater précisément ce suicide et encore moins la naissance de l'écrivain ; il est vrai que nous ne sommes guère mieux avancés aujourd'hui. Au moins sait-on maintenant que Des Périers naquit dans le village bourguignon d'Arnay-le-Duc et non à Bar-sur-Aube en Champagne («au Duché de Bourgogne » prétend cependant La Croix du Maine ; «en Bourgogne » écriront encore Bayle et Marchand), comme on l'a généralement cru jusqu'au milieu du XVIII ${ }^{\mathrm{e}}$ siècle. Le Dijonnais Étienne Tabourot fut le premier et longtemps le seul à dire Des Périers "Arnay le Duchois », dans ses Bigarrures (1583), il est vrai fréquemment rééditées jusqu'en $1660^{12}$.

Autre précision, plus importante : ceux qui avaient lu le traité Des scandales (1550) de Calvin avaient appris que Des Périers, tout comme Rabelais et Antonio de Gouvea, avait un temps «gousté l'Evangile» avant de s'en détourner et de sombrer dans la chiennerie antichrétienne ${ }^{13}$.

Un autre point sur lequel Du Verdier se montre original concerne son appréciation de l'ouvrage. En effet, alors qu'un consensus massif rassemblant catholiques et protestants accrédite la thèse d'un livre impie, «un Lucianisme, qui merite d'estre jetté au feu avec l'autheur, s'il estoit vivant », comme dira Étienne Pasquier (1586 et 1610) ${ }^{14}$, Du Verdier prétend tout le contraire. Lui a le livre en main, il le résume en détail et déclare n'y avoir rien décelé «qui merite d'avoir esté plus censuré que la Metamorphose d'Ovide, les dialogues de Lucian, \& les livres de folastre Argument \& fictions fabuleuses ${ }^{15} »$. Cette appréciation connaîtra une belle fortune au XVIII ${ }^{\mathrm{e}}$ siècle, après la reprise et les développements que lui donnera Prosper Marchand $(1711)^{16}$. Mais avant cela, au milieu du XVII ${ }^{\mathrm{e}}$ siècle, l'avocat et poète bon vivant Guillaume Colletet (1598-1659) l'adoptait dans sa vie manuscrite de Des Périers. Il avouait ne pas comprendre le jugement de Pasquier, ce grand connaisseur et admirateur de Rabelais, à propos de la «cymbale à sonnette resonnante » de Des Périers :

\footnotetext{
il me semble que c'est estre bien rigoureux et que c'est aller bien viste pour un docte et franc Gaulois, qui n'estoit pas tellement ennemy des vieilles hardiesses et de l'ingénieuse raillerie qu'il en voulut effacer tous les caractères dans les œuvres de Lucien mesme. Et si le Pantagruel de Rabelais luy a tant pleu, comme luy ont si souvent reproché ses adversaires, je m'estonne pourquoy il traitte si mal cette cymbale à sonnette resonnante, qui ne despleut pas aux curieux de son temps et qui fit alors tant de bruit. Il faut que j'advoue que les divers eschantillons que j'en ay veus dans la Prosopographie et dans
}

11 Jean Chassanion, Des grands et redoutables jugemens et punitions de Dieu advenus au monde..., Morges, Jean le Preux, 1581, chap. XXIV : «Des Epicuriens \& atheistes », p. 139-140 (rééd. 1586: Histoires memorables..., p. 140); La Croix du Maine, op. cit. («Il se tua enfin avec une espée...»); Nicolas Catherinot, L'Art d'imprimer, Bourges, s.l., 1685, p. 7-8; Mersenne, Colophon aux Quaestiones celeberimae in Genesim..., Paris, Cramoisy, 1623, dans la première version alléguée infra, n. 21 ; Guillaume Colletet, «Vie de Bonaventure Desperiers. $1542 », \mathrm{BnF}, \mathrm{Nafr} .3073$, f. $151^{\mathrm{r}}$; Simon Goulart, Histoires admirables ..., $2^{\mathrm{e}}$ éd., Paris, Jean Houzé, 1603, f. 78 ${ }^{\mathrm{v}}$ (puis Arras, La Rivière, 1604, p. 161 ; Cologne, Paul Marceau, 1610, p. 106-107 ; Genève, Samuel Crespin, 1620, p. 106-107 ; etc.).

12 Les Bigarrures du sieur Des Accords, Paris, Jean Richer, 1583, chap. XVII, p. 154 ${ }^{\mathrm{r}}$. Sur le lieu de naissance de Des Périers, voir Lionello Sozzi, Les Contes de Bonaventure Des Périers. Contribution à l'étude de la nouvelle française de la Renaissance, Turin, Giappichelli, 1965, p. 10-12.

13 Voir supra, n. 8.

14 Pasquier, Lettres, Paris, L'Angelier, 1586, VIII, p. $246^{\mathrm{r}}$ [à Tabourot] ; Thresor d'histoires admirables et memorables de nostre temps, Paris, Marceau, 1610, p. 106. Bayle citera ce jugement.

15 A. du Verdier, Bibliotheque, op. cit., p. 1177.

16 Éd. du CM, Amsterdam, P. Marchand, 1711, p. 30-32 et passim. 
la Bibliothèque de Du Verdier ne sont nullement impies ni criminels, et qu'il y a des dialogues dont le plus sévère Caton du Christianisme ne rougiroit point d'estre l'autheur ${ }^{17}$.

Colletet ajoute à cela un argument qui doit immédiatement retenir notre attention : «Avec tout cela je ne laisse pas de m'en rapporter aux esprits forts qui ont vu l'ouvrage entier et qui sont capables d'en porter un jugement sincère et désintéressé ${ }^{18} \gg$. Nous sommes placés là devant l'unique trace, au XVII ${ }^{\mathrm{e}}$ siècle, d'une lecture du Cymbalum mundi par des « esprits forts » contemporains, au reste bien difficiles à nommer, car Colletet en fréquenta bon nombre, soit dans l'entourage de Théophile (on sait que sa participation au Parnasse satyrique lui valut quelques tracas), soit plus tard dans celui de Naudé, son ami intime, et des autres «libertins érudits ${ }^{19}$ ». Que ces derniers aient jugé le Cymbalum inoffensif n'a rien $\mathrm{d}$ 'anodin et nous incite à ne pas regarder comme propre à leurs successeurs du XVIII siècle la condescendance culturelle consistant à ne s'imaginer aucun devancier véritablement dépris de la «superstition » religieuse, ni apte à pratiquer l'art d'écrire entre les lignes.

Le jugement pour le moins indulgent de Colletet et de ses amis resta néanmoins minoritaire au $\mathrm{XVII}^{\mathrm{e}}$ siècle. Mersenne, en 1623, nous donne l'exemple d'une lecture totalement contraire. Comme Du Verdier, comme les amis déniaisés de Colletet, le Minime semble avoir eu le livre en main (sans doute alors le même exemplaire), ou du moins disposer d'informations très précises à son sujet, mais à l'opposé de ces derniers il n'y découvre que des horreurs. Dans la première version - bientôt réécrite et dirigée contre un ennemi déiste jugé plus consistant ${ }^{20}$ - du colophon fameux «Contre les athées » qui concluait la première Quaestio des Quaestiones celeberimae in Genesim $(1623)^{21}$, il évoque «les pasquins (pasquilli) qu'à notre époque comme dans les siècles précédents certains athées et hommes impies font subrepticement circuler». Il mentionne alors une récente "Chronique de ceux à qui l'on dit que la fortune sourit », puis le plus ancien Cymbalum mundi qu' « un fripon d'une impiété achevée ${ }^{22} »$, «Bonaventura de Perez»-ne prendrait-il pas l'auteur pour un marrane ?-, a «traduit en français, afin que la France ne soit pas privée d'une telle monstruosité ». Beaucoup ont affirmé que ce de Perez était athée, ajoute Mersenne, en sorte que certains ont prudemment inscrit en tête de ses dialogues : Dixit insipiens in corde suo, non est Deus, et consigné qu'il eut la fin que méritait sa vie très impie. On voit par ces détails, et encore par l'affirmation que le Cymbalum fut donné en 1538, que Mersenne se référait à l'exemplaire de Pierre de L'Estoile aujourd'hui conservé à la Réserve de la Bibliothèque

17 Guillaume Colletet cité par Louis Lacour, Euvres françoises de Bonaventure des Périers, Paris, Jannet, 1856, t. I, p. LXIV, n. 1. Cette «Vie de Bonaventure Desperiers. 1542 »(date présumée de son décès) est extraite de la Vie des poètes françois de Colletet, manuscrit brûlé avec la Bibliothèque du Louvre en 1871 dont subsiste une copie partielle, «faite probablement pour Aimé Martin [1782-1847] », à la BnF : Nafr. 3073-3074 ; ici 3073, f. 150 sq., et notre citation au f. 152v . Jean-Claude Arnould et Bénédicte Boudou l'ont publiée en annexe à leur «Bibliographie d'Agrégation 2008-2009: Bonaventure des Périers, Nouvelles récréations et joyeux devis », Seizième siècle, n 5, 2009 (p. 319-341), p. 337-341; notre citation est à la p. 340.

18 J.-C. Arnould et B. Boudou, ibid. Voir aussi L. Sozzi, Les Contes..., op. cit., p. 72.

19 Voir René Pintard, Le Libertinage érudit dans la première moitié du XVII siècle, Paris, Boivin, 1943, passim (Index) ; et La Mothe Le Vayer, Gassendi, Guy Patin, Paris, Boivin, 1943, p. 24 : Colletet serait le Marcellus des Dialogues d'Orasius Tubero (1630-1633) de La Mothe Le Vayer.

20 Voir Claudio Buccolini, «Dal De Tribus impostoribus ai Quatrains du deiste : metamorfosi dell'ateismo nella doppia redazione del colophon di Mersenne », Bruniana \& Campanelliana, XIII-1, 2007, p. 169-177.

21 «Primae quaestionis adversus Atheos Colophon in quo Athei expugnandi modus affertur», dans Quaestiones celeberimae in Genesim..., Paris, Cramoisy, 1623, [col. 669-676 : deux feuillets], col. 669. Dans la seconde version («Primae quaestionis adversus atheos colophon : Deistarum impietas et errones aperiuntur atque reperiuntur »), il ne sera plus question du $C M$, non plus que dans les Paralipomena en fin d'ouvrage, qui accueillaient un sommaire du chapitre remplacé (col. 1829-1831); cependant Athei Bonaventura Cymbalum Mundi et Cymbalum Mundi demeureront dans l'index.

22 Ainsi Bayle traduit-il impiissimus nebulo. 
nationale (Rés. Z-2442), celui-là même sur lequel Prosper Marchand assiéra son édition en $1711^{23}$. Au reste, il décrit assez justement le contenu dudit Cymbalum :

Il se compose de quatre dialogues: dans le premier figurent Mercure, Byrphanes, Curtalius et l'Hotesse; dans le second Trigabus, Mercure, Rhetulus, Cubercus et Drarig; dans le troisième Mercure, Cupido, Celia, Phlegon, Statius et Ardelio. Dans le quatrième, enfin, sont introduits deux chiens parlants, Hylactor et Pamphagus. Des Périers attache à tous ces dialogues maintes fables sur Mercure, Jupiter, etc., au moyen desquelles il semble se rire de la foi catholique et rejeter tout ce que nous disons et croyons très vrai au sujet de $\operatorname{Dieu}^{24}$.

Mersenne, alors, ne manque pas de souligner la pernicieuse influence de Lucien sur Des Périers. Un peu plus loin, alors qu'il en appelle à la censure des mauvais livres susceptibles de propager «la mortalité de l'âme et d'autres erreurs qui peuvent conduire à l'athéisme », il mentionne l'affreux De tribus impostoribus ainsi que le livre de Vallée, puis de nouveau la fabellam Mercurii e coelo descendentis, «la fable de Mercure descendant du Ciel auprès de tous les hommes, qui sont comme des chimistes et chercheurs de la pierre philosophale, traduite du latin en français par l'impie Peresius, dans laquelle, si je ne me trompe, il insinue en ses trois dialogues [sic] que tout ce qu'on dit de la religion ne repose sur aucun fondement et doit être tenu pour des balivernes ${ }^{25}$. »

Bien que rare (on n'en connaît aujourd'hui que quatre exemplaires, dont celui de la BnF A 952 [1]), le réquisitoire de ce premier colophon contre les «athées » connaîtra une bien plus grande fortune critique que la seconde version anti-déiste ${ }^{26}$. Avant même que Prosper Marchand n'en diffuse largement le contenu dans sa «Lettre sur le Cymbalum Mundi » accompagnant son édition des quatre dialogues (1711), et bien avant ses rééditions dans la Bibliothèque Britannique (1743) et dans le Nouveau Dictionnaire historique et critique de Chaufepié (1753) ${ }^{27}$, Gilbert Voet (Voetius) en faisait état dans sa dissertation «De Atheismo » (1639), publiée dans la première partie de ses Selectarum Disputationum theologicarum (1648). C'est là que Bayle, qui insère dans la deuxième édition de son Dictionnaire historique et critique (1702) un article bien informé sur Des Périers - à ceci près qu'il reconnaît n'avoir jamais vu son Cymbalum -, va recueillir l'opinion de Mersenne à son

23 Cet exemplaire de 1538, marqué en tête «Delestoille » à l'encre rouge, comporte en effet, en haut du premier dialogue, la citation manuscrite du Ps. 12 (14), de la main de Pierre, selon Tom Hamilton (Pierre de L'Estoile and his World in the Wars of Religion, Oxford Univ. Press, 2017, p. 174) et, du même, sur la page de titre : «Telle vie, telle fin. Averé par la mort de ce miserable, indigne de porter le nom d'homme ». Une autre main a écrit sur cette même page : «L'auteur, Bonaventure Des Periers, homme meschant et athee, comme il appert par ce destestable livre. » Une supplique du libraire Morin au chancelier Du Bourg a été insérée dans cet exemplaire, «Ecritte de la main de M. Du Puy », suivant une copie que l'on trouve dans l'exemplaire de 1537 aujourd'hui à Versailles (voir F. Frank, éd. du CM, 1873, op. cit., p. XXIII-XXIV). Or, Pierre Dupuy était un ami de L'Estoile : peut-être avait-il hérité de son $C M$, avant que celui-ci ne rejoigne la collection royale (dont Dupuy et son frère Claude assurent la garde dès 1633). Dans cette hypothèse, c'est dans le Cabinet Dupuy, auquel Mersenne eut accès, ainsi que bon nombre de « libertins érudits », que le $C M$ était consultable.

24 Quaestiones..., col. 669 (« [...] fabulas [...] per quas fidem Catholicam irridere, et ea, quae de Deo verissima esse dicimus, et credimus, rejicere velle videtur ») ; la traduction est nôtre.

25 Ibid., col. 672 («[...] quicquid de religione dicitur, nullo fundamento niti, et pro nugis habendum »). Nous corrigeons «\& omnes homines », incompréhensible dans le texte, en « ad omnes homines » (voir notre note dans La Lettre clandestine, 26, 2018, p. 171-172). C'est évidemment par erreur que Mersenne, à cet endroit, évoque «trois dialogues ». De même, il suggère que le De tribus impostoribus pourrait être de Vallée « ou de quelque autre vaurien ».

26 Voir Buccolini, art. cit. n. 20.

27 P. Marchand, éd. cit., p. 22-23 et 34-38 (\$ 6: «Le Père Marin Mersenne ») et passim (voir les rééd. du CM en 1732, p. 26-28 et 41-46, et 1753, p. 24-26 et 37-42) ; Bibl. Brit., vol. XVIII-2, janvier-mars 1742, p. 408421 ; Jacques Georges Chaufepié, Nouv. Dict., Amsterdam et La Haye, Libraires associés, t. III, 1753, s.n. «Mersenne », p. 80-81. 
sujet, notamment sa suggestion que «des Ouvrages badins, \& pleins de fictions » peuvent véhiculer les idées les plus hardies ${ }^{28}$. La remarque n'était évidemment pas neuve, Calvin et Estienne l'avaient déjà formulée ${ }^{29}$, mais il faut croire que l'essor conjoint de la théologie rationnelle et de l'athéisme tout spéculatif qu'elle engendrait, l'avaient quelque peu éclipsée ${ }^{30}$.

Nous voici arrivés à la charnière du XVIII ${ }^{\mathrm{e}}$ siècle et nous ne pouvons clore ce chapitre sans rappeler qu'apparaîtra en Allemagne, entre 1692 et 1710, un pseudo-Cymbalum Mundi latin, traité spéculatif manuscrit qui, après la redécouverte du Cymbalum original, reprendra son titre initial de Symbolum sapientiae. Ce Cymbalum / Symbolum a fait l'objet d'une édition savante en 2000 et de plusieurs études ${ }^{31}$. Il n'a rien à voir avec la farce ignorée de Des Périers, si ce n'est sans doute l'orientation athée ${ }^{32}$, mais atteste à coup sûr de la curiosité qu'exerçait toujours le brûlot disparu de Des Périers.

Il n'est pas utile de nous étendre davantage sur ce point, ni sur la réception de l'authentique Cymbalum au XVIII ${ }^{\mathrm{e}}$ siècle : nous avons déjà rappelé ailleurs que ce siècle, qui était celui de la redécouverte du Cymbalum Mundi (et celui du jugement de la Sorbonne à son sujet), était aussi celui de sa plus grande occultation dans l'ordre intellectuel ${ }^{33}$. Sa tendance lourde, inaugurée par l'édition commentée de Prosper Marchand (1711), qui reprochera à Bayle de parler imprudemment d'un livre qu'il n'a pas vu et qui s'avère en réalité bien anodin, et entérinée entre autres par Goujet et Voltaire, sera en effet de n'y voir que de plaisantes fadaises non toujours compréhensibles, injustement condamnées par une Sorbonne et des magistrats aussi stupides que liberticides. Cette appréciation, nous l'avons déjà noté, consonne assez bien avec la lumineuse supériorité que le "siècle des Lumières " s'octroie volontiers sur les siècles obscurs, peuplés d'âmes plus grossières, aveugles et crédules, qui l'ont précédé. Quelques voix dissonantes s'élevèrent néanmoins au XVIII siècle, comme d'autres s'étaient élevées aux $\mathrm{XVI}^{\mathrm{e}}$ et $\mathrm{XVII}^{\mathrm{e}}$ siècles contre l'opinion majoritaire contraire (Du Verdier, Colletet et ses « esprits forts »), mais l'air du temps ne les ébruita guère.

Pendant un ample demi-siècle, incluant la Révolution et l'Empire, qui fait la charnière entre le $\mathrm{XVIII}^{\mathrm{e}}$ et $\mathrm{le} \mathrm{XIX}^{\mathrm{e}}$ siècles, il n'est presque plus question du Cymbalum - sinon, furtivement, dans quelques dictionnaires et catalogues de vente -, mais les choses vont ensuite radicalement changer. Dans le contexte général de cette restauration de la littérature du $\mathrm{XVI}$ e siècle qui s'amorce autour de $1820^{34}$, et sous l'impulsion particulière de deux hommes de lettres, l'érudit Éloi Johanneau (1770-1851) et le mieux connu Charles Nodier (1780-1844), le Cymbalum Mundi va résolument et durablement s'inscrire sous le signe d'une réhabilitation à

28 Pierre Bayle, Dict. hist. crit., Amsterdam, Reinier Leers, 1702, t. II, s.n. «Periers (Bonavanture des)», p. 2380-2381, spéc. la Rem. B, p. 2381. Sylvain Maréchal reprendra cette appréciation dans son Dictionnaire des athées anciens et modernes, Paris, Grabit, an VIII [1800], s.v. «Periers (Bonavanrture des) », p. $334 ; 2^{\mathrm{e}}$ éd., Bruxelles, s.n., 1833, p. 210.

29 Voir par ex. R. Estienne, à propos des «mocqueurs de Dieu» et notamment du $C M$ : « Sçavons-nous pas que le but de ceux-ci a esté, en faisant semblant de chasser la melancholie des esprits et leur donner du passetemps [...] [de] venir apres [...] donner des coups de bec à la vraye religion Chrestienne ?» (Traité preparatif, éd. Boudou, XIV, op. cit., p. 292-293).

30 Sur cette forme d'athéisme post-spinoziste, on verra bien sûr Gianluca Mori, L'Ateismo dei moderni. Filosofia e negazione di Dio da Spinoza a d'Holbach, Roma, Carocci Editore, 2016.

31 Cymbalum mundi sive symbolum sapientiae, éd. Guido Canziani, Winfried Schröder et Francisco Socas, Milan, Franco Angeli, 2000. Schröder fait le point bibliographique sur ce texte dans « Un "spinozisme de gauche" : le Symbolum sapientiae », La Lettre clandestine, 26, 2018 (p. 69-79), p. 69-70.

32 Nous avons notamment soutenu cette position philosophique dans « Le livre de Jupiter. L'athéologie du Cymbalum mundi », Bibliothèque d'Humanisme et Renaissance, LXXVIII-1, 2016, p. 333-361.

33 Voir A. Mothu, «Règlement de comptes à Amsterdam autour du Cymbalum mundi (1711 sq.) », La Lettre clandestine, 24, 2016, p. 255-285.

34 Sur la «vague seiziémiste » alors en pleine expansion, voir Jacques-Rémi Dahan, Introduction à Ch. Nodier, Études sur le seizième siècle et sur quelques auteurs rares ou singuliers du dix-septième, Bassac, Plein Chant, 2005, p. 32 sq. 
la fois littéraire et philosophique. Cette période faste de la réception du Cymbalum a déjà été sommairement étudiée ${ }^{35}$, bornons-nous à en compléter le tableau de quelques précisions.

C'est incontestablement à Nodier que revient la palme de la revalorisation littéraire du Cymbalum. Dans un article très influent de novembre 1839 sur «Bonaventure Desperiers », plusieurs fois réédité après sa parution dans la Revue des deux mondes ${ }^{36}$, il élève l'auteur au grade de «talent le plus naïf, le plus original et le plus piquant de son époque » et sacre son Cymbalum 1' « un des chefs-d'œuvre de langue du seizième siècle » ${ }^{37}$. D'autre part, il soutient que ces derniers dialogues ne sont ni si opaques, ni si inoffensifs que l'avaient voulu croire Voltaire - ennemi intime de Nodier - et avec lui la majeure partie du XVIII ${ }^{\mathrm{e}}$ siècle lettré.

Sur ce point, Nodier avait bien sûr quelques devanciers, mais il avait surtout un contemporain : Éloi Johanneau, récent éditeur de Montaigne, Charron et surtout Rabelais ${ }^{38}$. Ce dernier avait servi de prétexte à leurs premiers échanges au début des années 1820 et le Cymbalum mundi, dont Nodier possédait un exemplaire de 1538, nourrira de nouveaux échanges à la fin de la décennie.

C'est en mars 1829 que Johanneau révèle au bibliothécaire de l'Arsenal ce qu'il appelle alors sa «clef » du Cymbalum mundi, soit une découverte dont il a commencé à tirer tout un faisceau d'explications des quatre dialogues qu'il rassemble dans une Clef du Cymbalum mundi. Cette découverte, déjà évoquée, consiste dans le décryptage anagrammatique des noms de Thomas du Clevier et Pierre Tryocan, dont il conclut que l'auteur du Cymbalum est un incrédule cherchant à « évangéliser » un ami croyant.

Nodier se montra jaloux de cette découverte a priori si simple, il chercha à s'en emparer et finit, en désespoir de cause, par affirmer qu'il l'avait faite à l'âge de 15 ans sans s'aviser alors de sa valeur ni songer à la breveter. Cette affaire si pénible pour sa mémoire est plutôt bien connue, mais il nous importe de signaler qu'il existe depuis 2005 aux Archives départementales du Loir-et-Cher, à Blois, un dossier Johanneau qui contient quantité d'informations inédites ayant trait au sujet ${ }^{39}$. On y trouve, entre autres, diverses épreuves de la fameuse Clef de Johanneau - qui sera publiée en 1841, sous la forme d'une Lettre au baron de Schonen, dans l'édition Lacroix du Cymbalum mundi et autres œuvres de Bonaventure Des Périers $^{40}$; une copie manuscrite partielle du Cymbalum et des notes préparatoires à une

35 Voir Verdun-Louis Saulnier, «Le sens du Cymbalum mundi de Bonaventure des Périers » (1), BHR, XIII-1 (1951), p. 43-69, spéc. p. 43-46 ; François Roudaut «La Réception du Cymbalum mundi, 1538-1824 », et Guy Bedouelle, «Le Cymbalum mundi au XIX siècle », dans Franco Giacone (dir.), Le Cymbalum Mundi (Actes du colloque de Rome, 3-6 novembre 2000), Genève, Droz, 2003, respectivement p. 77-102 et 129137.

36 Charles Nodier, «Bonaventure Desperiers », Revue des deux mondes, $4^{\mathrm{e}}$ s., t. XX, $1^{\mathrm{er}}$ nov. 1839, p. 329351 ; réédité à Bruxelles la même année, puis en 1841 dans Bonaventure Desperiers, Cirano de Bergerac (Paris, Techener), ensuite en tête de l'édition Jacob des Contes ou Nouvelles récréations (Paris, Gosselin), et plusieurs autres fois au cours du siècle. Voir sa réédition moderne par J.-R. Dahan dans les Études sur le seizième siècle... de Nodier, op. cit., avec la note bibliographique p. 412. Laurent Calvié a également réédité ce texte dans son édition modernisée du CM (Toulouse, Anacharsis, 2002, p. 141-174.

37 Cette dernière citation n'est pas dans la version originale de l'article de Nodier, mais dans un alinéa ajouté en 1841 (voir l'édition des Contes citée ci-dessus, p. 13); on y lit «quinzième siècle », leçon évidemment fautive que nous corrigeons, à la suite de J.-R. Dahan (éd. cit., p. 206).

38 Sur la vie et la carrière de cet érudit blésois, nommé en 1830 Conservateur des monuments d'art des résidences royales, voir avant tout Bernard Edeine et Léopold Cabanau, «Éloi Johanneau (1770-1851). Un savant solognot méconnu », Mémoires de la Société des Sciences et Lettres de Loir-et-Cher, t. XLIX, 1994, p. 111-148. Pour Rabelais, nous évoquons l'édition dite « Variorum » réalisée avec Charles Esmangart, Paris, Dalibon, 1823-1826, 9 vol.

39 Voir l'inventaire de ce fonds sur http://archives.culture41.fr/archive/fonds/FRAD041_1_J/view:17646. Nous avons examiné ce dossier en mars 2017 et en rendons partiellement compte dans « Nodier, Johanneau et le Cymbalum mundi. L'éclairage d'un dossier blésois » : https://hal.archives-ouvertes.fr/hal-01888517

40 Paul Lacroix (alias le bibliophile Jacob) éd., Le Cymbalum mundi et autres auvres de Bonaventure des Périers [...]. Avec une Lettre à M. de Schonen, contenant une Clef du Cymbalum, Paris, Charles Gosselin, 
édition critique apparemment entamée en 1832; une correspondance avec divers savants touchant notre texte, et en particulier les difficultés à en publier la Clef en 1829 en raison de la censure monarchiste $^{41}$; des pièces rassemblées par l'auteur pour démontrer éventuellement devant les tribunaux que Nodier s'était indûment approprié sa découverte. Signalons enfin l'exemplaire original, corrigé et augmenté, d'une plaquette satirique rarissime visant Nodier : Charles Nodier mis au Panthéon charivarique de sa découverte de la clef du Cymbalum mundi à l'âge de 15 ans. Pièce qui sera multigraphiée et distribuée à des relations avant d'être publiée, presque identique, dans le Bulletin du bibliophile d'août $1847^{42}$.

Sitôt parue, la Clef de Johanneau ne cessera de perdre en estime ${ }^{43}$. Lui appliquant à peu près les mêmes griefs qu'à l'édition Variorum de Rabelais, on reprochera à l'auteur son esprit de système et son souci maniaque de tout interpréter au prix d'explications souvent hasardeuses. Devant l'Histoire, il est incontestablement le père de ces «thèses de la hardiesse » du Cymbalum (ainsi que les appelle Saulnier), qui allaient se succéder au fil du siècle, notamment dans les éditions et rééditions données par Paul Lacroix, Louis Lacour et Félix Frank, avec toutefois des variantes tenant à l'idéologie propre à chacun ${ }^{44}$. Comme ce dernier le disait justement, Johanneau «s'égare souvent dans le détail », mais il a au moins «discern(é) la tendance maîtresse ${ }^{45}$ ». Toutefois c'est bien Nodier, le premier disciple malgré lui de Johanneau, qui donnera le ton, malgré les erreurs et approximations multiples qu'on lui reprochera. L'autorité que lui confère à l'époque sa haute position sociale, intellectuelle et médiatique, ainsi que son style élégant et «passionné », comme dira encore Frank ${ }^{46}$, expliquent cet ascendant, non moins que sa lecture quelque peu brumeuse ou élastique de l'hétérodoxie du Cymbalum («impiété », «scepticisme railleur»), ouverte à plusieurs

1841, p. 77-154 : Lettre de M. Éloi Johanneau à M. le baron de Schonen, ou Clef du Cymbalum mundi (datée, à la fin, du 12 mars 1829).

41 Nous consacrons une note à ce sujet dans « Jésus et le bon vin, et autres notes sur le Cymbalum mundi», La Lettre clandestine, 26, 2018, p. 179-183.

42 Aux p. 359-361. J.-R. Dahan signale un échantillon de ce Panthéon dans la collection d'autographes Allard du Chollet à la BnF, ms. Nafr. 24010, f ${ }^{\circ} .354$ (éd. Nodier, Études sur le seizième siècle, op. cit., p. 413, note 1). Nous n'avons pas examiné cet exemplaire que Dahan dit «multigraphié ». Notons, comme Dahan, que le Bulletin du bibliophile de juillet 1849 évoquera encore l'affaire Nodier-Johanneau (J.G.A.L., «Clef du Cymbalum mundi », p. 243-247, ici 246-247). Nous reproduisons l'original de Blois dans notre article cité supra n. 39.

43 Notons cependant que c'est pour des raisons conjoncturelles qu'elle ne sera pas rééditée dans la seconde édition Jacob de 1858 : Lacroix explique dans l'Avertissement qu'il n'a pas été autorisé à reproduire ce «morceau curieux, quoique rempli de paradoxes inadmissibles» (p. VI de l'éd. de luxe; p. 5 de l'éd. commune). Dans sa première édition, déjà, il notait qu'elle valait surtout par l'ingénieux décodage de la dédicace mais «touche à l'exagération d'un système philosophique qui appartient tout entier à M. Éloi Johanneau » (p. VII).

44 Saulnier, «Le sens du Cymbalum mundi », art. cit., p. 45 ; Max Gauna parle à leur sujet d'une quête des «précurseurs courageux de l'anticléricalisme français » («Pour une nouvelle interprétation du Cymbalum mundi », La Lettre Clandestine, 6, 1997, p. 157-172, ici p. 163). L'édition Lacroix [bibliophile Jacob] de Paris, Delahays, 1841, sera remaniée en 1858 (ibid.), puis reprise à l'identique par Garnier frères en 1872 et jusqu'au milieu du XX $\mathrm{XX}^{\mathrm{e}}$ siècle. L'édition Lacour, donnée dans les Euvres françaises de Des Périers (Paris, Jannet, 1856), sera reprise, corrigée et allégée en collaboration avec Damase Jouaust, en 1874 (Paris, Librairie des bibliophiles). L'édition Frank (Paris, Lemerre, 1873) ne connaîtra aucune réimpression. La séquence 1872-1874 serait-elle politiquement signifiante? Nous ne sommes pas sûr que le bibliothécaire Louis Lacour (1832-1892), le plus retenu de tous dans l'interprétation du Cymbalum (Des Périers serait un rationaliste modéré demeuré théiste, voire panthéiste), fût un républicain convaincu (juste après l'écrasement la Commune, il réclamera que lui soit restitué le patronyme noble de La Cour de La Pijardière, que sa famille portait avant la Révolution). Par contre, le poète, critique littéraire et érudit prolifique Félix Frank (18371895) semble affectionner l'image d'un Des Périers à la fois irréligieux, voire athée, et contestataire politique. Les frères Haag, dans la $2^{\mathrm{e}}$ édition de leur France protestante, en retiendront «l'effrayante hardiesse » de la pensée de Des Périers (t. V, 1886, p. 361).

45 Éd. Frank, op. cit., p. XLII.

46 Ibid., p. XXXV. 
possibles, depuis le déisme sincère niant seulement la divinité du Christ (Lacour) jusqu'à l'expression militante d'un "contre-évangile » intégral (Frank), en passant par l'extrémisme satirique rattaché aux «idées nouvelles de la Réforme » (Lacroix $)^{47}$.

Cependant ce ne sont pas là encore les éléments les plus décisifs. Il faut souligner que Nodier avait amarré le Cymbalum à une vie. Il avait campé un personnage, une psychologie, et pas seulement un livre ou des idées. De Des Périers, il avait brossé le portrait d'un être à part, un authentique «génie incompris » : celui d'un artiste total, poète, musicien, prosateur sans égal, dont les écrits comme la vie « sont enveloppés d'un profond mystère »; celui d'un excentrique assez comparable à Cyrano de Bergerac (Nodier les associera dans une brochure de 1841), dont le Cymbalum mundi révèle qu'il fut aussi un homme du refus et que son destin lui échappa, tant et si bien qu'il mit peut-être fin à ses jours : «Jamais fatalité plus obstinée ne s'est attachée à la réputation d'un auteur et de ses écrits ${ }^{48} \gg$. Un héros romantique, en somme.

Alain Mothu
(Sorbonne-Université, CELLF 16-18)

47 En 1858, du moins, car dans sa $1^{\text {ère }}$ édition de 1841, Lacroix se montrait plus proche des idées radicales de Johanneau.

48 Ch. Nodier, «Bonaventure Desperiers » (1839), art. cité, p. 335 ; nos citations précédentes : p. 329 et 333. Nodier reste flou au sujet du suicide de Des Périers (p. 330-331). 